\title{
Wie viele Ärzte braucht das Land?
}

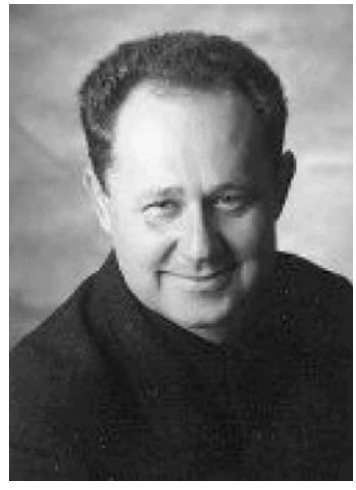

Prof. Dr. Manfred Wildner
Bibliografie

DOI http://dx.doi.org/

10.1055/s-0031-1277149

Gesundheitswesen 2011;

73: 209-210

(c) Georg Thieme Verlag KG Stuttgart · New York ISSN 0941-3790

Korrespondenzadresse Prof. Dr. M. Wildner

Bayerisches Landesamt für Gesundheit und Lebensmittelsicherheit Veterinärstraße 2 85762 Oberschleißheim Manfred.Wildner@lgl.bayern.de
Das Schauspiel für die anwesenden Gäste muss eindrücklich gewesen sein: kaum hatte Ludwig XIV., der „Sonnenkönig“, ein Glas Wein getrunken, sprudelte die Hälfte des Getrunkenen zu seiner Nase wieder heraus. Grund war ein Defekt im Gaumen - eine unerwünschte Komplikation der Maßnahme seines Leibarztes, ihm alle Zähne zu ziehen, zur größeren Glorie Frankreichs und seiner Majestät. „Zum Zweck der Desinfektion habe ich seiner Majestät das Loch im Gaumen 14-mal mit einem glühenden Eisenstab ausgebrannt“, notiert Dr. Daquin einen Monat später [1]. Waren doch nach unbestrittener Lehre der Sorbonne, der Dr. Daquin entstammte, die Zähne die gefährlichsten Infektionsherde des menschlichen Körpers. Ob die vereinten Bemühungen seiner Leibärzte Dr. Vallon, Dr. Faggon und Dr. Daquin seiner Gesundheit mehr Schaden als Nutzen zugefügt haben, sei dahingestellt. „L'Etat, c'est moi!“ (dt. „Der Staat bin ich!“) - dem absolutistischen Selbstverständnis des großen Monarchen haben seine Leiden keinen Abbruch getan.

Ob dieses Modell der Leibärzte (gr. Archiatros, „Chefarzt“), welche rund um die Uhr zur Verfügung stehen, tatsächlich ein Optimum an medizinischer Versorgung gewährleistet, gar ein Optimum an Gesundheit? Das tödliche Ringen König Ludwig II. von Bayern mit seinem Psychiater Dr. Gudden im Wasser des Starnberger Sees weist ebenfalls auf möglicherweise gesundheitsschädigende (Leib-)Arzt-Patienten-Interaktionen. „Ich brauche keine Ärzte, die mir meine Laster verbieten, sondern solche, die sie mir ermöglichen!“, soll ein römischer Kaiser gesagt haben. Dieses Diktum ist wiederum erhellend für eine Medizin, welche sich von der therapeutischen Notwendigkeit entfernt und sich zunehmend zu einer wunscherfüllenden Medizin wandelt.

Im Licht des Sozialgesetzbuches (SGB) verbietet sich derartiges Agieren, zumindest auf Kosten der Solidargemeinschaft. § 70 SGB V bestimmt: „Die Krankenkassen und die Leistungserbringer haben eine bedarfsgerechte und gleichmäßige, dem allgemein anerkannten Stand der medizinischen Erkenntnisse entsprechende Versorgung der Versicherten zu gewährleisten. Die Versorgung der Versicherten muss ausreichend und zweckmäßig sein, darf das Maß des Notwendigen nicht überschreiten und muss in der fachlich gebotenen Qualität sowie wirtschaftlich erbracht werden". Die in den geschilderten historischen Fällen anzuwendenden Urteile wären Über- und Fehlversorgung, mangelnde Evidenzbasis und fehlende Wirtschaftlichkeit. In wieweit zusätzlich vermeidbare (Kunst-)Fehler zu thematisieren wären, ist eine weitere Frage. Eine mögliche Schlussfolgerung:
Mehr ist nicht unbedingt besser, auch nicht bei der ärztlichen Versorgung.

Doch auch ein zu wenig an ärztlicher oder psychotherapeutischer Versorgung schadet mit großer Sicherheit der Gesundheit. Richtig ist, dass sich der Erhalt der Gesundheit und die Heilung bzw. Linderung von Krankheit komplementär, nicht identisch zu einander verhalten. Mehr als die Hälfte der in den letzten hundert Jahren sich verdoppelnden Lebenserwartung ist überwiegend auf Public Health/Hygiene-Maßnahmen u.a. Entwicklungen zurückzuführen, nicht auf individualmedizinische Leistungen. Demgegenüber ist der Großteil der verbesserten Überlebenschancen und Lebensqualität bei Krankheit und Verletzungen von der kurativen und rehabilitativen medizinischen Versorgung bewirkt.

Der Bedarf an haus- und fachärztlichen Leistungen wird aufgrund demografischer Entwicklungen in Deutschland hoch bleiben und aufgrund einer Zunahme des Machbaren wie des Gewünschten wohl weiter steigen. Etwa 430000 Ärztinnen und Ärzte waren im Jahre 2009 bei den Landesärztekammern gemeldet, was einer jährlichen Steigerungsrate von knapp $2 \%$ entspricht. Nur jeder dritte Arzt war berufstätig (ca. 325000), zunehmend auch in Teilzeit, bei steigenden Ärztinnenzahlen: Ärztinnen haben einen Anteil von knapp $44 \%$ an der Gesamtzahl und knapp $60 \%$ bei den Erstmeldungen bei den Ärztekammern. Dieser „Feminisierungstrend“ ist bei den Studienanfängern weiter steigend.

Von den verschiedenen Verbänden und Körperschaften wird auf stagnierende bzw. in Relation sinkende Hausärztezahlen bei gleichzeitig steigenden Fachärztezahlen verwiesen - ein europaweiter Trend. Das Saldo an verfügbaren Ärzten ist im ambulanten Sektor jedenfalls positiv. Und auch im stationären Sektor finden sich steigende Arztzahlen - Folge einer Leistungsverdichtung, welche in Deutschland zu höheren Zahlen an Krankenhaus-Behandlungsfällen trotz sinkender Bettenzahlen und Liegezeiten geführt hat. Dass bei steigenden Arztzahlen insgesamt und vielfach geschlossenen kassenärztlichen Versorgungsgebieten das Durchschnittsalter in den Teilkompartimenten Krankhaus (,ich kann mich (noch) nicht niederlassen“) und in der Konsequenz auch in der ambulanten Versorgung steigt, ist zunächst ein nüchtern erwerbsbiografisch erklärbares Phänomen. In einem System kommunizierender Röhren dürfte sich ein freiwerdender Kassenarztsitz rasch wieder besetzen lassen wenn er denn attraktiv ist. Auf unbesetzte Kassenarztsitze insbesondere in ländlichen Regionen wird von den Kassenärztlichen Vereinigungen 
und Ärztekammern immer wieder mahnend hingewiesen. Von den Ersatzkassen wird demgegenüber darauf verwiesen, dass 800 fehlenden Ärzten in wenigen Regionen etwa 25000 überzählige Ärzte in überversorgten Regionen gegenüberstehen [2]. Hier ist eine differenzierte Diskussion angezeigt, wie sie auch z.B. von der Weltgesundheitsorganisation unterstützt wird [3]. Lässt sich daraus die Notwendigkeit steigender Ausbildungskapazitäten in der Humanmedizin ableiten, gar ein absehbarer Ärztemangel? Der Medizinische Fakultätentag (MFT) äußert sich hierzu dezidiert ablehnend. Eine vom MFT veranlasste Sonderauswertung des Statistischen Bundesamtes zu Absolventenzahlen belegt deutliche Ausbildungssteigerungen der Medizinischen Fakultäten und Höchstzahlen beim medizinischen Nachwuchs, der auch überwiegend (94\%) klinisch tätig werden will [4]. Die Quote von 90\% erfolgreichen Studienabschlüssen ist eine Bestnote verglichen mit dem bundesweiten Durchschnittswert von $72,5 \%$ bei allen Studiengängen. Ein vom Bundesgesundheitsministerium in Auftrag gegebenes Gutachten (Ramboll-Gutachten) stellt fest, dass unter den Wechslern in verschiedene Führungs- und Managementfunktionen im Gesundheitswesen und andere Public Health-Bereiche über die Hälfte die klinische Tätigkeit erst nach der fachärztlichen Weiterbildung aufgeben [5]. Studien, die sich mit den genannten Daten nicht decken, sollten wegen wahrscheinlicher methodischer Mängel mit Zurückhaltung aufgenommen werden [4].

Wiederum statistisch liegt die Ärztedichte in Deutschland im Schnitt der entwickelten Industrienationen sehr hoch - mit 3,3 Ärzten /100000 Bevölkerung deutlich höher als beispielsweise in Japan (2 Ärzte/100 000 Bevölkerung) mit seiner weltweit führenden Lebenserwartung. Umgekehrt lässt sich die hohe Ärztedichte in Griechenland (4,3 Ärzte/100000 Bevölkerung) nicht mit einer hervorragenden Platzierung bei der medizinischen Versorgung korrelieren. Was ist die „richtige“ Ärztedichte und gibt es diese überhaupt? Bei einer ökonomischen Betrachtung ist von einer großen Elastizität der Nachfrage nach ärztlichen Leistungen über einen Grundbedarf hinaus auszugehen, also einer grundsätzlich nach oben offenen Nachfrage, welche stark vom Preis bestimmt wird. Legen diese länderspezifischen Durchschnittswerte nicht starke kulturelle und systembedingte Einflüsse auf den Bedarf bzw. auf Angebot und Nachfrage nahe? „L'Etat, c'est nous!“ (dt. „Der Staat sind wir!“), ist das große Freiheitsversprechen unserer demokratischen Grundordnung einschließlich der Freiheit festzulegen, wie viele Ärzte/innen oder Psychotherapeuten/innen wir wollen.

Darf sich die Diskussion dabei an Absolutzahlen und Durchschnittswerten erschöpfen? „Am Durchschnitt ist die Kuh ersoffen“, sagt ein statistisches Bonmot, welches darauf hinweisen will, das beruhigende Durchschnittswerte nichts über die mögliche Unbill extremerer Ausprägungen sagen. So wie eben plötzliche tiefe Stellen eines im Durchschnitt flachen Dorfteichs möglich und dann auch verhängnisvoll sind. Worauf uns dieses Bonmot hinweisen will, ist ein Verteilungsproblem („Fehlallokation“) der kostbaren Ressource Arzt/Ärztin und anderer Gesundheitsberufe. Die Anreize auf die Verteilung werden zum Teil monetär gesetzt. Eine Studierendenbefragung der Universität Trier macht darüber hinaus deutlich, dass auch Standortfaktoren großen Einfluss auf die Berufsentscheidungen haben: Orte mit weniger als 5000 Einwohnern werden gemieden, die Vereinbarkeit von Familie und Beruf hat mit $96 \%$ bei den Einflussfaktoren den höchsten Stellenwert [6].

Damit sind die mit den Steuerungsaufgaben betrauten Institutionen gefragt. Vorhandene Personalressourcen - in den über- versorgten Gebieten und bei den nicht berufstätigen Ärzten/ Ärztinnen - lassen sich durch geeignete Anreize vermutlich in größerem Maßstab zurückgewinnen und im Interesse der Bevölkerungsgesundheit steuern. Denkbare Maßnahmen sind flexible Praxismodelle mit Angestelltenverhältnissen, Teilzeitanstellungen, Kinderbetreuungen für Arbeitnehmer/innen an Krankenhäusern, Studienplatzquoten für Niederlassungen in ländlichen Bereichen, Vergütungsangleichungen vor- und „nachklinischer“" (Management-) Bereiche mit dem klinischen Bereich, familiengerechte Dienstzeiten und Organisationsformen, finanzielle Anreize durch Bereitstellung von Praxis- und Wohnräumen, die Freisetzung ärztlicher Kapazität durch die Verringerung der „Bürokratiekosten“ und durch neue Gesundheitsberufe wie Gemeindeschwester/-pfleger, Stationssekretären/innen und Operationsassistenten/innen, E-Health-Unterstützung im ländlichen Raum, Anreize für eine zum Patienten gehende („ambulante“) und sprechende Medizin. Die Frage lautet nicht nur, wie viele Ärzte das Land braucht, sondern auch wo und mit welchen Qualifikationen. Die Ärzteschaft sollte hierzu Antworten mit der gebotenen (Public Health-)Expertise als Beitrag zur Steuerung von Prozessen in komplexen Systemen geben können.

Solchen Steuerungsfunktionen und anderen Gesundheitsfragen gehen die Beiträge in diesem Heft nach: sozialer Ungleichheit und der Qualität der Arzt-Patienten-Beziehung, regionalen Unterschieden der Lebenserwartung und vermeidbarer Sterbefälle, der tabakattributablen Mortalität, Gesundheitswirkungen der Prävention und Auswirkungen des Stalkings.

Dass auch Leibärzte solchen Systemfragen offen gegenüber stehen können, belegt Francois Quesnai (1694-1774). Zunächst als Wundarzt chirurgisch ausgebildet, richtete er eine Streitschrift gegen die Ansicht des damaligen Leibarztes des Königs Ludwig XV., Krankheiten freizügig mit Aderlässen zu behandeln. Seine weitere Karriere führte ihn, nach erfolgreicher Behandlung der Windpocken des Thronfolgers und mit Unterstützung durch Madame Pompadour schließlich selbst zur Anstellung als königlicher Leibarzt. In dieser Position überwand er die berufliche Abgrenzung der schneidenden und nicht-operativ tätigen Ärzte. In höherem Alter entwickelte Quesnay Interesse an Fragen der Volkswirtschaft. 1758 entwickelte er ein Modell des wirtschaftlichen (Geld-)Kreislaufes, vermutlich orientiert an seinen Kenntnissen über den Blutkreislauf. Wesentlicher Fortschritt war die Erkenntnis, dass Ausgaben nicht nur verbrauchte Ressourcen sind, sondern an anderer Stelle als Einnahmen erscheinen und damit eine Interdependenz der Wirtschaftsprozesse bedingen. (Auch) solche Ärzte braucht das Land.

\section{Literatur}

1 Zander HC. Napoleon in der Badewanne. Das Beste aus Zanders Großer Universalgeschichte. LIT Verlag, Zürich 2004; S. 97-S. 108

2 Ersatzkassen fordern Abbau der Überversorgung. 20.01.2011. URL: http//:www.aerzteblatt.de/nachrichten/4436 Zugriff am 21.01.2011

3 World Health Organization. Models and tools for health workforce planning and projections. Human Resources for Health Observer 3. WHO, Genf; 2010

4 Bitter-Suermann D. Ärzteschwund/Ärztemangel: wo liegen die Probleme? Forschung und Lehre 2011; 18 (1): 42-44

5 Bundesministerium für Gesundheit und Soziale Sicherung. Gutachten zum „Ausstieg aus der kurativen ärztlichen Berufstätigkeit in Deutschland“. Hamburg, Ramboll Management 2004, URL: http://www. ratgeber-versand.de/files/ratgeber/51/f-335_291_6513f4.pdf Zugriff am 05.04.2011

6 Berufsmonitoring Medizinstudenten 2010, URL: http://www.kbv.de/ publikationen/37141.html Zugriff am 04.04.2011 
Hinweis:

Dieser Beitrag hat folgenden Fehler im Text.

\section{Erratum}

Zum Editorial "Wie viele Ärzte braucht das Land" (Gesundheitswesen 2011; 73: 209-210) DOI: 10.1055/s-0031-1277 149 wird um Beachtung folgender Korrekturen gebeten:

Richtig hätte es heissen müssen, dass nur etwa drei von vier Ärzten/innen berufstätig sind. Richtig hätte der Nenner beim Ländervergleich “je 1.000 Einwohner" lauten müssen. Die zugehörigen Zahlen einer älteren OECD-Statistik beziehen sich auf "praktizierende Ärzte" (siehe zur Definition http://stats.oecd.org/glossary/detail.asp?ID=2099). Der Begriff "practising physician" ist anders definiert als unsere Kategorie der "berufstätigen Ärzte", was der internationalen Vergleichbarkeit geschuldet ist. Diese Verhältniszahlen lassen sich nicht direkt aus den im Editorial genannten Arztzahlen errechnen. Aktuelle Werte (Bezugsjahr 2008 in der OECDStatistik 2010; URL: http://www.oecd.org/document/46/ 0,3343,en_2649_34631_34971438_1_1_1_1,00.html, Zugriff 05.05.2011) sind 3,5/1.000 für Deutschland, 2,2/1.000 für Japan und 6,0/1.000 für Griechenland. 